\title{
Historia del presente: hacer bizarro lo evidente
}

\author{
"History of they present. Turning the self-evident bizarre"
}

\author{
Ana Grondona \\ analuciagrondona@gmail.com \\ CONICET - Instituto de Investigaciones Gino \\ Germani - Centro Cultural de la Cooperación Floreal \\ Gorini, Argentina
}

Cita sugerida: Grondona, A. (2021). Historia del presente: hacer bizarro lo evidente. Sociohistórica, 47, e129. https://doi.org/10.24215/18521606e129

\begin{abstract}
Resumen: El artículo propone algunas reflexiones sobre el uso de la historia desde una genealogía del presente. Para ello, se organiza en tres ejes. En primer lugar, se exponen algunas consideraciones sobre los modos en que desde esta perspectiva se trabaja sobre la temporalidad de ciertos problemas; en segundo lugar, retomando algunas preguntas vinculadas con la cuestión de la "traducción" y la "circulación", se abordan las maneras en que lidia con su dimensión espacial; finalmente, se reflexiona sobre la historia del presente como forma de la crítica y sus criterios de rigurosidad. A lo largo del texto, y con el fin de ilustrar o clarificar algunos aspectos conceptuales, se retoman ejemplos de investigaciones previas que, inspiradas en la historia del presente, indagaron sobre el problema de la pobreza en el discurso experto, por una parte, y en el de la cuestión racial en el discurso sociológico argentino, por la otra.
\end{abstract}

Palabras clave: Genealogía, Crítica, Sur, Temporalidades.

Abstract: The paper reflects upon the use of history within a genealogy of the present. To do this, it is organized into three sections. In the first place, we propose some considerations on the ways in which this perspective understands temporality; Secondly, taking up some questions related to the issue of "translation" and "circulation", we look into the ways in which it deals with the spatial dimension; finally, the paper elaborates on the history of the present as a form of criticism and its validation criteria. Throughout the text, and in order to illustrate or clarify some conceptual aspects, we present examples from previous investigations that, inspired by the history of the present, studied the problem of poverty in the expert discourse, on the one hand, and of the racial question in the Argentine sociological discourse, on the other.

Keywords: Genealogy, Critique, South, Temporalities.

El ejercicio de la historia del presente (Dean, 1994; Garland, 2014; Walters, 2012) se propone una eventualización (Foucault, 1995) o rarificación de ciertas evidencias (Veyne, 1984) para preguntarse por las condiciones y los modos en que determinada práctica, institución, concepto, problema o tecnología (por poner algunos ejemplos) llegó a ser tal como se nos aparece en un momento determinado (sea o no contemporáneo al de la indagación). En este sentido, retoma la invitación de Michel Foucault (1995) a una investigación histórico-filosófica capaz de conocer el "pasado de las verdades" antes que las "verdades del pasado”. Para ello, se vale de una estrategia arqueo-genealógica de trabajo con documentos, interesada 
en regularidades y discontinuidades discursivas y de historizar las disputas de saber-poder que atraviesan determinados regímenes de lo visible y lo enunciable.

Estas indicaciones, muy generales, suelen quedar más claras a partir del concepto de problematización según lo han trabajado Michel Foucault (2001), Robert Castel (2001) y Mitchell Dean (1994), así como de la noción de problemática acuñada por Louis Althusser y Étienne Balibar (2004). Según las definiciones que proponen estos autores, "problematizar" no equivale a representar un objeto preexistente, ni a crear por el discurso un objeto que no existe (Revel, 2008, p. 70), sino a hacer entrar algo en el juego de lo verdadero y lo falso, es decir, convertirlo en objeto del pensamiento (Foucault, 2001). La problematización, como haz de interrogantes, ensambla elementos de diversa procedencia, cuyo sentido está sobredeterminado por dicha articulación. Una sociología de las problematizaciones (Haidar, 2013), interesada en cuestiones como el desempleo, la pobreza, la marginalidad, el desarrollo, la domesticidad, los riesgos del trabajo, etc., debe ser, entonces, capaz de describir estos elementos y de dar cuenta de sus diversas procedencias, sus combinaciones y mutaciones (Castel, 2001). Al decir de Étienne Balibar, la genealogía se hace “por elemento”, lo que supone una indagación dispuesta a descomponer analíticamente aquello que se presenta ya-articulado, es decir, poder diferenciar ciertos conceptos, determinadas técnicas de observación y medición, tales orientaciones prácticas, ciertos tonos y estilos de argumentación, determinados modos de presentar o legitimar una voz autorizada, etc. En este mismo sentido, otro concepto clave es, justamente, el de ensamblaje, que, según Victoria Haidar alude a

un conjunto de elementos o componentes que se encastran entre sí produciendo un cierto efecto de conjunto. Ese ensamblaje no es nítido ni preciso; contrariamente, involucra superposiciones, solapamientos y ejercicios de resistencia por parte de algunas piezas que se niegan a encastrarse con otras (Haidar, 2008, p. 248).

A partir de estas coordenadas, aún muy abstractas, en las páginas que siguen expondremos algunas reflexiones sobre el uso de la historia desde una genealogía del presente. Para ello nos centraremos en tres ejes. En primer lugar, nos detendremos en algunas consideraciones sobre los modos en que desde esta perspectiva se trabaja sobre la temporalidad de ciertos problemas; en segundo lugar, retomando algunas preguntas vinculadas con la cuestión de la "traducción" y la "circulación", nos referiremos a las maneras en que lidia con su dimensión espacial; finalmente, reflexionaremos sobre la historia del presente como forma de la crítica y sus criterios de rigurosidad. A lo largo del texto, y con el fin de ilustrar o clarificar algunos aspectos conceptuales, retomaremos ejemplos de investigaciones previas que hemos emprendido bajo la inspiración de la historia del presente alrededor del problema de la pobreza en el discurso experto (Grondona, 2014), por una parte, y de la cuestión racial en el discurso sociológico argentino (Grondona, 2019c), por la otra.

\section{Historias DESALINEADAS}

La invitación a trabajar a partir de las nociones de problematización y ensamblaje se diferencia nítidamente de cualquier intento de reconstruir la narrativa o el despliegue histórico-evolutivo de determinado problema social. Por una parte, la mirada de la historia del presente no pretende "reponer" un estado de cosas del pasado; por la otra, no es el hilo de una narración lo que resulta de este ejercicio, sino más bien un patchwork de elementos heterogéneos y muchas veces contradictorios. Encuentra, en este punto, afinidades con otras perspectivas, como la de Didi-Huberman (2011), interesadas en disolver la evidencia del "contexto" para poner a jugar las múltiples temporalidades y asincronías que configuran nuestros objetos. También encontramos resonancias con el modo en que el Análisis Materialista del Discurso trabaja sobre la historicidad compleja y sobredeterminada de los enunciados a partir de la noción de Interdiscurso (Glozman y Montero, 2010), sobre la que volveremos más abajo.

En virtud de ello, los corpora que se componen inspirados por la historia del presente no respetan las cronologías establecidas por el estado del arte, ni se dejan llevar por la presunción sobre la linealidad del 
tiempo. Se parecen más a un rejunte abigarrado de distintas piezas discordantes. Lejos de ser un punto de partida, en el proceso de indagación se va generando, como resultado parcial o final, un corpus conformado por distintas "series" con diferentes criterios de organización (Foucault, 2002). Dado que el ejercicio de rarificación es producto de un proceso, a contramano de lo que sugiere buena parte de los manuales de metodología, sería imposible partir de una delimitación de los materiales con los que se va a trabajar, e incluso de las variables que se tomarán en cuenta para su selección. Presuponerlas, supondría jugar dentro de los límites de lo "ya sabido" y contribuir a la reproducción de las narrativas del campo. Si bien la indagación se inicia (incluso sin saberlo) a partir de formas de estructuración de los documentos más próximas a aquellas con las que los problemas sociales "se presentan", en su decurso se producen modos de ordenamiento que desestabilizan la evidencia de esas organizaciones primeras.

El resultado de este decurso será un corpus multitemporal. El concepto de Interdiscurso de Michel Pêcheux (2012), autor central del Análisis Materialista del Discurso, resulta inspirador a este respecto. Según este autor, "la condición esencial de la producción y de la interpretación de una secuencia no es inscribible en la esfera individual del sujeto psicológico: ella reside de hecho en la existencia de un cuerpo sociohistórico de huellas discursivas" (Pêcheux, 2012, pp. 145-146, énfasis nuestro). Esto supone reconocer la historicidad específica de los discursos, que no puede reducirse a la del "contexto inmediato" en el que una secuencia se formula: ${ }^{1}$ si tomamos en serio la metáfora según la cual una secuencia o formulación es resultado de un proceso de producción, debemos preguntarnos por las condiciones que la determinan, las que genera, pero también aquellas con las que se encuentra. Estas condiciones son heterogéneas, incluyen entramados institucionales, encrucijadas epocales, trayectorias individuales, etc. Asimismo, hay determinaciones vinculadas específicamente a la materialidad de lo que "puede y debe decirse", de "lo ya dicho" que retoma una secuencia (a espaldas y más allá de la conciencia de sus productores). Precisamente, el "término Interdiscurso caracteriza ese cuerpo de huellas como materialidad discursiva, exterior y anterior a la existencia de una secuencia dada, en la medida en que esa materialidad interviene para constituirla" (Pêcheux, 2012, pp. 145-146, énfasis nuestro). Así, el sentido que se pone en juego en cualquier documento no se produce "al interior" del texto ni del individuo (o los individuos) involucrado(s) en su formulación, sino en su relación con otros que retoma, niega, con los que se antagoniza. Estas relaciones (inter)discursivas conforman una dimensión clave de las condiciones de producción de una secuencia y están asociadas a la heterogeneidad constitutiva que rige cualquier decir (Aguilar, Glozman, Grondona y Haidar, 2014).

Desde esta perspectiva, en una investigación que se inicia a partir de la delimitación de ciertas secuencias o series de referencia lo que habrá serán, entonces, hipótesis respecto de algunas huellas o trazos y su vínculo con otras zonas del Interdiscurso. En virtud de ello, se desplegarán remisiones a determinadas secuencias previas, simultáneas o posteriores, en virtud de las que se delinearán dominios interdiscursivos ${ }^{2}$ en los que resulta relevante indagar para entender algunas de las condiciones históricas que intervinieron en la producción de las secuencias o series de referencia (a su vez asociadas a un cierto modo en que se presenta una problemática o problematización, a un cierto ensamblaje de elementos, a una pregunta por alguna de sus singularidades).

Por cierto, las investigaciones tienen una instancia previa a poder definir las series o secuencias de referencia: una exploración a tientas, en la que luego de una gran dispersión y de extraviarnos en un recorrido a veces caprichoso e inútil, encontramos algo inquietante o inesperado o recurrente (según los casos) que llama nuestra atención y provoca una pregunta. Puede ser un detalle. Por ejemplo, la autonarración de un experto que afirma que antes de dedicarse a los estudios de pobreza se dedicaba a asuntos "totalmente distintos" y refiere con ello a su trabajo en el Plan Trienal del tercer gobierno de Juan Perón. Frente a ello, lejos de "asumir la perspectiva de los actores", como recomiendan algunas sociologías, se dispara la pregunta sobre si resulta tan "evidente" que se trató de discusiones "distintas”, sin relación entre sí. Muñidos de esa inquietud, la lectura de ciertos documentos, más allá de los explícitamente referidos por los textos con los que iniciamos nuestra pesquisa, y de los límites espacio-temporales con los que iniciamos nuestro trabajo sobre saberes expertos contemporáneos de la pobreza, encontramos que en el Plan que "nada tenía que ver" con los asuntos 
de nuestro interés puede leerse: "este es un plan de liberación. Liberación de las necesidades básicas de los argentinos, cuya satisfacción les será asegurada, cualquiera sea su actividad o lugar en que vivan" (Presidencia de la Nación, 1973, p. 3, énfasis nuestro). Ante esta formulación, que tensa la hipótesis de discontinuidades con la que el experto en cuestión se narraba a sí mismo, comenzará una exploración que pondrá en relaciones documentos que no se habían leído articuladamente.

Del mismo modo, podemos extrañarnos ante la presencia de un documento sobre las razas firmado por un organismo internacional en un manual para estudiantes del primer año de sociología. Nuevamente, nos enfrentamos a la pregunta sobre el régimen de evidencias en virtud de la cual tal texto pudo convivir con otros (de Herskovitz o Lipton o Germani) cuyo género describiríamos hoy como "académico". También la insistente repetición de ciertas formulaciones puede llamar nuestra atención: la tematización de la ética del trabajo y la empleabilidad en los documentos del Plan Jefes y Jefas de Hogar Desocupado, por caso. A partir de ella podríamos interrogarnos sobre la "obvia" responsabilidad individual frente a la condición de "sin trabajo" en el marco de una tasa de desempleo de casi $20 \%$.

Tal como indicamos más arriba, en este primer extrañamiento el texto, fragmento o documento en cuestión (donde simplemente "pescamos" algo, para echar mano de la jerga psicoanalítica), funciona como secuencia de referencia (Aguilar et al., 2014). Una vez establecida (precaria y preliminarmente), comienza otra etapa de dispersión que, mediante remisiones a partir de marcas textuales y regularidades, nos llevan de un documento a otro, de un conjunto de documentos a otros. Una marca textual puede aparecer como "rareza", como un elemento inesperado o disonante que llama inmediatamente nuestra atención. Sin embargo, un "hallazgo" muchas veces viene precedido de una cierta monotonía (algo así como la carta robada de Poe que "estaba ahí"), porque no solo importa encontrar "tal" documento o "determinada" formulación, sino estar en condiciones de verlo/leerlo efectivamente.

A lo largo del proceso de trabajo los materiales suelen organizarse en distintos conjuntos. Pueden estar agrupados de acuerdo con criterios "epocales", "institucionales" o según se trate de distintas dimensiones, aspectos o modos de interrogación de la problematización analizada. Los primeros criterios suelen ser los más comunes al comienzo de la indagación, que sólo cerca del final puede aproximarse a un ordenamiento producto de lo que Althusser llama "un conocimiento"3 (2004).

Quizás resulte clarificador presentar un ejemplo basado en una investigación en curso sobre los modos en que aparece problematizada la cuestión racial en los textos de Gino Germani. En ella delimitamos (luego de varias etapas de indagación, dispersión y reformulación) una organización de los materiales del corpus en cinco series: (1) una serie de referencia, con un conjunto de textos del sociólogo (incluía clases, textos "de puño y letra", traducciones, ediciones, etc.); (2) un conjunto de documentos asociados a las políticas raciales del régimen italiano entre 1935 y 1939; (3) materiales vinculados con los debates de la agrupación antifascista Giustiziae Liberta alrededor de las políticas raciales del régimen fascista italiano entre 1936 y 1939 ; (4) textos que recogen las discusiones sobre el problema racial en el marco de UNESCO en la segunda posguerra 1946-1955 y (5) documentos en los que se observa una tematización de lo racial en los albores de la sociología argentina entre 1884-1915.

En una primera instancia, la disposición respondió a criterios más bien clásicos y "obvios". Sin embargo, en función de la pregunta y el proceso de investigación, el corpus que sedimentó como su resultado se fue organizando según dimensiones o aspectos vinculados a la problemática analizada y sus mutaciones. Así, podemos pensar en una serie de fragmentos alrededor del debate sobre si pueden o no delimitarse distintas razas y cuáles son o deberían ser; otra sobre el problema del mestizaje y sus consecuencias, una más con fragmentos que definen o se interrogan sobre quiénes están habilitados para discutir sobre estas temáticas, del mismo modo, una a propósito del vínculo entre esos saberes expertos y lo que resulta generalizado como saber del sentido común, otra sobre la relación entre lo heredado y lo adquirido, finalmente, una sobre la in/existencia de relación entre determinantes biológicas, mentalidades políticas y predisposición hacia la democracia o el autoritarismo, etc. De alguna forma, el trabajo de investigación es el camino entre ambos 
modos de organización y disposición de los materiales. Mientras la primera parte de ciertas delimitaciones espacio-temporales más o menos "dadas", la segunda presenta una combinación heterogénea de tiempos a partir de criterios y periodizaciones que resultan pertinentes en virtud del análisis de los propios documentos y de cierta problematización, de modos que poco tendrán que ver con la imagen de una "línea del tiempo".

\section{HiSTORIAS DESCENTRADAS Y CONTAMINADAS}

A diferencia de las academias de los países centrales, quienes investigamos en el Sur, solemos privilegiar objetos de "nuestros propios contextos". Sin embargo, a poco andar, descubrimos que los fenómenos que han suscitado nuestro interés (el problema de la pobreza, los modos en que la sociología argentina tematizó la cuestión racial, las racionalidades involucradas en el gobierno de las poblaciones sin trabajo, etc.) están atravesados por múltiples procesos de traducción y circulación. Nuestras ciudades, nuestras lenguas, nuestros modos de vestir, nuestras políticas sociales, nuestros saberes expertos son (im)puro cocoliche. Bien mirado, esta es la "regla" para cualquier "contexto", pero en nuestros objetos periféricos las voces del centro suelen estar marcadas: se refieren a tal innovación francesa, a tal autor austríaco, se validan en esos señalamientos de autoridad y combinan, desprolijamente, lo que en el centro ha permanecido "por separado". Producimos traducciones bizarras que también nos permiten ver a contraluz lo paradójico de lo que se presenta como la versión "clásica". Esta colocación estructural habilita, como punto de mira, a preguntarse por las singularidades de la parte, del todo y de la relación que los une.

Tanto como la multitemporalidad a la que referimos en el apartado anterior, la "politopia" o "pluriespacialidad" también recorre nuestras investigaciones. Sin embargo, las narrativas (e incluso las genealogías críticas) producidas desde el Centro suelen denegarla, desatendiendo las huellas o trazos que en sus objetos de análisis remiten a memorias vinculadas con otros territorios. Las preguntas por la circulación o traducción de ciertas prácticas, técnicas, racionalidades o diagnósticos suelen privilegiar el sentido NorteSur o Centro-Periferia.

Los estudios de Ortiz (1973) sobre la transculturación -originalmente publicados en 1940- son un antecedente fundamental para dar cuenta de la politopia de los fenómenos que estudiamos, así como de las relaciones de fuerza que borran sistemáticamente la productividad del Sur. Del mismo modo, la introducción de Questions of modernity (2000) abona a nuestro argumento. En ese libro, Mitchell retoma una diversidad de trabajos históricos que muestran que dispositivos, saberes, discursos que Foucault asociaba a "Occidente" pueden ser objeto de genealogías contaminadas que, al descentrarse de Europa, hacen visibles procedencias no-occidentales: la racionalización del trabajo en las plantaciones del Caribe antes que en Mánchester; el Panóptico in nuce desarrollado por Samuel Bentham en la colonización rusa del territorio Otomano antes que su hermano Jeremy; el sistema lancasteriano de enseñanza en Bengala antes que en Europa; la pregunta por la población antes en las colonias que en la agenda de los fisiócratas europeos; la literatura inglesa como unidad que se configura para/en la socialización de los niños de la India; y una lista abultada de etcéteras. Este es también el sentido de la indagación de Eduardo Grüner sobre la Revolución haitiana y los modos en que ella resonó en la dialéctica de Hegel (más allá de las intenciones del filósofo alemán). Al respecto, como saldo teórico-epistemológico, Grüner nos invita a:

Instalarnos (epistemológica pero al mismo tiempo ideológica y políticamente, puesto que esas cosas son inseparables) en la perspectiva de una tensión, de un conflicto no resuelto entre la Parte y el todo, en la cual lo que se juega es la cuestión del poder y la dominación, es una manera de estar además, desde el lugar de la construcción de un saber no sometido a la colonialidad ("construcción", subrayamos, porque el saber no está hecho de una vez para siempre: no es una mera acumulación de conocimientos "neutrales”, sino un permanente campo de batalla) (Grüner, 2010, p. 55).

Tal como sugiere la cita precedente, hay un privilegio en el punto de vista de la Parte -es decir, de las periferias o del Sur-. Pero esta singularidad no nos coloca "fuera" de la historia del capitalismo, ni fuera de 
la modernidad, sino en torsiones o modulaciones en las que lo canónico se muestra bizarro. La discusión acerca del barroco latinoamericano también giró alrededor de esta misma cuestión: lo barroco como marca "fundacional" de la Conquista, como traza ineludible y "momento constitutivo" (Zavaleta, 1990, pp. 180 y ss.) que se reactualiza de diversos modos, también como lenguaje de la contra-Conquista. ${ }^{4}$ Un cierto modo de estar en la ambivalencia, en una transición en suspenso que horada las fantasías del tiempo lineal y progresivo. Una forma de experimentar lo insólito, lo extraordinario devenido cotidiano, lo anti-modélico, lo contraclásico que produce armonía en la desarmonía de vectores y tendencias opuestas que, en lugar de anularse, se refuerzan mutuamente.

Lo barroco como inspiración, como modo de experimentar el mundo y relación con la verdad, permite, en este sentido, un nuevo rodeo alrededor de las preguntas de la historia del presente, capaz de descentrarlas de su matriz eurocéntrica. Así, la inquietud por las procedencias múltiples e impuras se dispone no sólo a desordenar tiempos y ritmos, sino a descentrar también espacialmente al "yo" cartesiano, que todavía se esconde en las narrativas que insisten en ningunear (para volver sobre una palabra con la que a Grüner le gusta jugar) el papel de las periferias en las historias del Centro. Esta proyección de un Occidente homogéneo que se autoproduce y se despliega debería ser siempre objeto de eventualización por parte de una historia del presente que se pretenda crítica.

Una vez más, quizás resulte ilustrativo referirnos al ejemplo de la genealogía sobre los saberes de la pobreza en Argentina en la que la tematización de las necesidades básicas (insatisfechas) se mostró relevante (Grondona, 2014). Según supimos a partir de esa indagación, cuando a comienzos de la década de 1980 la construcción de ese tipo de indicadores para dar cuenta del creciente problema de la pobreza se generalizó en la región, los documentos y manuales locales señalaban como antecedente trabajos elaborados por OIT (en particular PME, 1976) o por CEPAL (Altimir, 1978). Por su parte, también las narrativas actuales sobre los estudios de la pobreza filian la cuestión en aquellas discusiones (Spicker, Álvarez Leguizamón y Gordon, 2009, pp. 208 y ss.) y, en algunos casos, incluyen aportes de la psicología, en particular de Abraham Maslow (tal el caso de la entrada de Wikipedia). Sin embargo, al seguir la estela de esos documentos y sus referencias llegamos, sin demasiada demora, a los escritos de Fundación Bariloche alrededor del Modelo Mundial Latinoamericano y la los de Oscar Varsavsky sobre "estilos de desarrollo" desplegados en el marco del Centro de Programación Matemática y, antes de eso, en el Centro de Estudios del Desarrollo de Venezuela.

En esta pesquisa encontramos un conjunto de documentos que, producidos en y desde América Latina, ponían en cuestión tanto las visiones optimistas del desarrollo como las críticas pesimistas que, por aquellos años (1969-1981), ya formulaban propuestas de decrecimiento; por caso, en el estudio impulsado por el Club de Roma sobre los "límites del crecimiento" (Grondona, 2016). Un conjunto de investigadores provenientes de las ciencias "duras" (geología, química, física, computación, matemática) intentaban demostrar mediante el uso de modelos matemáticos corridos por computadoras la viabilidad física, política y social de otros estilos de desarrollo que, en lugar de propender hacia el consumismo propio de los países desarrollados, estuvieran orientados a la satisfacción de las necesidades humanas básicas, entendidas de un modo muy amplio (desde las alimentarias, hasta las emocionales o cognitivas).

Por cierto, los vínculos entre aquellas discusiones y las referencias que el campo de los saberes expertos de la pobreza señala como procedencia del concepto de necesidades básicas son bastante inmediatos, pues, por ejemplo, en el caso de la OIT, Hugo Scolnik (codirector del Modelo Mundial Latinoamericano), participó -con un paper especialmente solicitado para el evento- de la reunión que definiría la posición del organismo en 1976. CEPAL, por su parte, fue ámbito de resonancia de las discusiones sobre estilos de desarrollo (especialmente, de los aportes de Oscar Varsavsky) al menos desde comienzos de la década de 1970 (vgr. CEPAL, 1971).

Ahora bien, aun cuando sea posible reconducir la lectura hasta estas discusiones, la operación de olvido (o ninguneo) en virtud de la cual median otras referencias no resulta inocua. En la "retraducción" de la cuestión de las necesidades básicas, según se presentó a comienzos de la década del ochenta (vgr. INDEC, 1984), esta 
quedó asimilada a la problemática de la pobreza. Esta superposición estaba en tensión con los debates según se habían desarrollado una década antes. Tal como indica el estado del arte que propone Oscar Altimir (1978) el uso del concepto de pobreza correspondía a "las políticas más conservadoras de mitigación de la pobreza y el uso del concepto de necesidades básicas con las estrategias más radicales de reorientación de desarrollo y de reorganización del orden social” (1978, p. 22, énfasis nuestro).

Este ejemplo (y podríamos citar otros, como los "antecedentes" chilenos del workfare que se presume noratlántico; ver Grondona, 2012) ilustra el argumento sobre la productividad de una historia del presente que, en virtud de su ánimo de desestabilización de las evidencias, no sólo impulse indagaciones interesadas en la heterogeneidad temporal que atraviesa a nuestros objetos, sino que también a sus múltiples geografías (y geopolíticas), particularmente en el caso de las voces subalternas y periféricas, sistemáticamente denegadas.

\section{HisTORIAS CRÍTICAS}

En reflexiones tardías, Michel Foucault inscribió su proyecto intelectual de una ontología del presente en la herencia de la crítica kantiana, una filosofía interesada en las condiciones de su presente. Este "ethos filosófico" es lo que delimitó como "actitud de modernidad":

La ontología crítica de nosotros mismos no hay que considerarla como una teoría, una doctrina, ni tampoco como un cuerpo permanente de saber que se acumula; es necesario concebirla como una actitud, un ethos, una vida filosófica en la que la crítica de lo que nosotros somos es, a la vez, análisis histórico de los límites que nos son impuestos y prueba de su posible transgresión (Foucault, 1995, pp. 17-18, énfasis nuestro).

En relación con el apartado anterior, y retomando los términos de Roberto Schwartz, sumaríamos que, en tanto la posición periférica delimita un escenario en que se despliegan conceptos, tecnologías, dispositivos que se muestran como "fuera de lugar", una historia del presente desde aquí trae la promesa de una mirada menos inclinada a tomarse literalmente aquello que se presume como ya-dado:

Lo que en Europa sería una verdadera hazaña de la crítica, entre nosotros podía ser el simple descreimiento de un cualquiera, para quien utilitarismo, egoísmo, formalismo o lo que fuere, son como unos atuendos entre otros, muy de moda, pero innecesariamente ajustados. Se ve que este sustrato social repercute en la historia de la cultura: una gravitación compleja, donde constantemente reaparece una constelación en la que la ideología hegemónica de Occidente hace el ridiculo, como una mania entre manias (2014, p. 195, énfasis nuestro).

Pues bien, este modo de entender la práctica de investigación de nuestros objetos y la relación con sus pasados conlleva una serie de preguntas asociadas a sus criterios de rigurosidad: si la interrogación por la historia no interesa en términos de una "reconstrucción" más o menos "ajustada", ni se orienta por la consigna de "completar" lo que hasta aquí habrían sido miradas sesgadas, sino de la desestabilización táctica de aquello que se presenta como sabido, no podrían ser parámetros de "correspondencia" los que orienten normativamente nuestra indagación. En este punto, resulta fundamental retomar seriamente las resonancias bélicas en juego, pues ellas remiten al presupuesto de que, antes que una ciencia que se acerca o se aleja de descripciones objetivas y fidedignas, lo que hay son luchas por producir la verdad, que conforman y sedimentan ciertos sentidos. No hay, entonces, posibilidad de un saber/verdad no posicionado. En virtud de ello, para una perspectiva crítica, como la que propone una historia del presente, es central entender esa relación de fuerzas (en el propio campo) e intervenir en ella. Ella se conjuga, siempre, con una coyuntura más amplia atravesada por ciertas contradicciones. La pregunta por ese estado de tensiones y los modos en que ciertas preguntas de investigación se articulan en ella es un aspecto nodal de la rigurosidad para una indagación inspirada en las coordenadas teóricas que hemos presentado someramente.

$\mathrm{Al}$ respecto, encontramos sugerente la figura althusseriana de "curvar el bastón", con la que intentó explicar sus pecados juveniles de "teoricismo" (Althusser, 2008, p. 218). La insistencia sobre el papel de la Teoría buscaba contrarrestar un cierto estado de la discusión intelectual en el que el empirismo era predominante 
y tenían efectos dogmáticos. En un sentido más general, el autor niega la existencia de una línea verdadera por la cual la filosofía materialista discurriría, aun cuando ese discurrir suceda como si la estuviera buscando constantemente (Cortés, 2019). En términos del autor francés:

Esta lucha, incluso para aquellos que han visto claro de antemano, se desenvuelve sin ninguna instancia que juzgue y dirima desde lo alto, es preciso hablar aquí, paradójicamente, de sin verdad y de desviación sin norma. Simplemente, este alejamiento no dominado, esta transgresión, esta aberración, esta derrota o esta crisis que lenta o repentinamente se manifiesta en lo real, sin verdad ni norma: he aquí el error y la desviación (Althusser, 2008, pp. 253-254).

En relación con este punto, y a contramano de algunas afirmaciones en las que Foucault (2001) desestimaba su valor intelectual, la polémica puede funcionar como punto de partida de una genealogía del presente. Por ejemplo, en el caso de la citada investigación sobre los modos en que aparece tematizada la cuestión racial en la sociología de Gino Germani, buscábamos discutir con las lecturas que lo presentan como un "empresario académico" (si aceptamos esa figura, deberíamos agregar que debió declararse en quiebra por lo menos dos veces) o simple promotor de la sociología estadounidense hegemónica, pues ellas tendían a invisibilizar trazos de sus textos que lo ligan a un conjunto de discusiones del antifascismo (como, agudamente, intuyó su hija; Germani, 2004). Yendo incluso contra la autopresentación que el sociólogo ítalo-argentino gustaba proyectar, la composición de un corpus de fragmentos a partir de la pregunta por "lo racial" subraya la dimensión política de sus escritos. Incluso de la disputa por la pretendida neutralidad valorativa, pues encontramos que la "ciencia", en particular alrededor de la cuestión racial (su in/existencia, las formas y grados de su determinación o de su plasticidad, etc.) eran un escenario de lucha, un campo a reconquistar. ${ }^{5}$ Así, leer algunos tramos de la sociología germaniana en relación con aquellas controversias, permite desestabilizar la homogeneidad con la que suele leerse su “impulso modernizador" y, en términos más generales, discutir algunos presupuestos que marcan cierto "clima de época" actual, impregnado por el giro "decolonial" que, en muchos de sus exponentes, lleva a subestimar la historia de los debates latinoamericanos. Por el contrario, la puesta en valor de esas discusiones (incluso en sus contribuciones presuntamente más "mainstream" como las de Prebisch o Germani), indagando en sus tensiones y heterogeneidades, nos permite volverlas contemporáneas y nos sustrae de la posibilidad de ponernos "por encima" o "por fuera" de sus interrogaciones.

En este proceso de desestabilización polémica es probable que encontremos elementos que maticen o relativicen nuestras hipótesis en virtud de otras marcas textuales. Incluir también esa complejidad, aunque debilite nuestro argumento, es otro modo de intentar una práctica rigurosa: no anteponer conclusiones (que de ninguna manera es lo mismo que "partir como una hoja en blanco"), sino alojar la heterogeneidad de elementos sin construir narrativas que los homogenicen (aunque sea bajo la forma de una contranarrativa). No se trata de "completar" estados del arte, sino de dispersar unidades dadas de antemano hasta desnaturalizarlas (la época, el autor, la escuela, el concepto).

Este ejercicio tendrá, sin embargo, sus límites. En tal sentido, aunque nos interesan los traspiés, las contradicciones y las tensiones presentes en los documentos con los que investigamos, pues nos permiten eludir las preguntas sobre las intenciones de sus "productores", sería riesgoso presumirnos más astutos que ellos. Nuestros textos también están gobernados por condiciones de producción, en buena medida invisibles para nosotros mismos (a esto aludía Pêcheux con la noción de Olvidon. ${ }^{\circ} 1$ ). Un asunto que no se resuelve "reflexivamente", como querría cierta sociología, pues las condiciones sobre las que podemos volver o repensar (Olvidon. ${ }^{\circ}$ ) no son todas las que nos determinan. Esta precariedad resulta, pues, insalvable y no se reduce a que las futuras generaciones "continúen" la faena, por el contrario, denuncia esa hipótesis de completitud como pura ilusión.

En virtud de las reflexiones que nos propusimos hilvanar en este texto, resulta claro que la pregunta por los "usos de la historia" que inspira este dossier interpela muy especialmente a una perspectiva interesada en la dimensión estratégica de los regímenes de saber-poder, que hace tiempo ha abandonado ilusiones sobre una 
"verdad" que vendría a aquietar las conciencias y las luchas y que parte, por el contrario, de que estas últimas han sido -y serán- incesantes.

\section{ReFERENCIAS}

Aguilar, P., Glozman, M., Grondona, A. y Haidar, V. (2014). ¿Qué es un corpus? Revista Entramados y Perspectivas, 4, 35-64.

Aguilar, P., Fiuza, P., Glozman, M. y Grondona, A. (2015). Hacia una genealogía del "Buen Vivir": Contribuciones desde el Análisis Materialista del Discurso. Theomai, 32, 96-127.

Althusser, L. (2004). La Revolución Teórica de Marx. Me\#xico: Siglo XXI.

Althusser, L. (2008). Defensa de tesis en la Universidad de Amiens. En La soledad de Maquiavelo (pp. 209-248). Madrid: Akal.

Althusser, L. y Balibar, E. (2004). Para Leer El Capital. México: Siglo XXI.

Altimir, O. (1978). La dimensión de la pobreza en América Latina. Cuadernos de la CEPAL, 27. Santiago de Chile: CEPAL.

Castel, R. (2001). Presente y genealogía del presente: Pensar el cambio de una forma no evolucionista. Archipiélago: Cuadernos de crítica de la cultura, 47, 67-75.

Comisión Económica para América Latina CEPAL (1971). Un modelo para comparar estilos de desarrollo o politicas económicas optativas. Santiago de Chile: CEPAL.

Cortés, M. (2019). Schmitt para las izquierdas: notas sobre marxismo y política. Trans/Form/Ação [online], 42(2), 151-164.

Courtine, J. (1981). Quelques problèmes théoriques et méthodologiques en analyse du discours, à propos du discours communiste adressé aux chrétiens. Langages, 62.9-128.

Dean, M. (1994). Sociology, Foucault and the uses of History. En Critical and Effective Histories: Foucault's Methods and Historical Sociology Critical and Effective Histories (pp. 13-42). NY: Routledge.

Didi-Huberman, G. (2011). Ante el tiempo. Historia del arte y anacronismo de las imágenes. Buenos Aires: Adriana Hidalgo Editora.

Foucault, M. (1995). Crítica y Aufklärung. Qu'est-ce que la Critique. Revista de Filosofía-ULA, 8, 1-18.

Foucault, M. (2001). Polémique, politique et problématisations. En Dits et écrits II. 1976-1988 (pp. 381-390). Paris: Gallimard.

Foucault, M. (2002). La arqueología del saber. Buenos Aires: Fondo de Cultura Económica.

Garland, D. (2014). What is a "history of the present"? On Foucault's genealogies and their critical preconditions. Punishment \& Society, 16(4) 365-384.

Germani, A. (2004). Gino Germani: del antifascismo a la sociología. Buenos Aires: Taurus

Glozman, M. y Montero, A. (2010). Lecturas de nunca acabar: consideraciones sobre la noción de interdiscurso en la obra de Michel Pêcheux. Cadernos de Letras da UFF, 12, 75-96.

Grondona, A. (2012). "Workfare" y "underclass", las singularidades de la traducción argentina. Revista Estudios del Trabajo, 41, 77-113.

Grondona, A. (2014). Saber de la pobreza. Discursos expertos y subclases en la Argentina 1956-2006. Buenos Aires: Ediciones CCC.

Grondona, A. (2019a). ¿Qué es el contexto? En P. de Marinis, Exploraciones en teoría social: ensayos de imaginación metodológica (pp. 227-268). Buenos Aires: CLACSO/IIGG. En prensa.

Grondona, A. (2019b). Ethos moderno y ethos barroco. Torsiones para una historia del presente desde el Sur. XIII Jornadas de sociología UBA.

Grondona, A. (2019c). Cuestión racial y sociología argentina: Sarmiento, Ayarragaray, Bunge e Ingenieros frente a Germani. Aportes en clave genealógica de cara al Sur. Revista de discursos y prácticas, 8(12), 3-32. 
Grondona, A. (comp.) (2016). Buen Vivir y estilos de desarrollo. Buenos Aires: Ediciones del CCC.

Grüner, E. (2010). La Parte y los Todos. Sobre algunas cuestiones preliminares. En La oscuridad y las luces (pp. 41-98). Buenos Aires: Edhasa.

Haidar V. (2013). La historia como condición para la inteligibilidad del presente: una aproximación desde la sociología de las problematizaciones. En XIV Jornadas Interescuelas/Departamentos de Historia. Departamento de Historia de la Facultad de Filosofía y Letras. Universidad Nacional de Cuyo, Mendoza.

Haidar, V. (2008). Trabajadores en riesgo. Buenos Aires: Prometeo.

INDEC (1984). La pobreza en la argentina. Indicadores de necesidades básicas insatisfechas a partir de los datos del censo nacional de población y vivienda 1980. Buenos Aires: INDEC.

Mitchell, T. (2000). The Stage of Modernity. En Questions of Modernity (pp. 1-34). Londres: University of Minnesota Press.

Ortiz, F. (1973). Contrapunteo cubano del tabaco y el azúcar. Barcelona: Ariel.

Pêcheux, M. (2012). Leitura e memória: Projeto de Pesquisa. En Análise de Discurso. Textos escolbidos (pp. 141-150). Campinas, SP: Pontes [Traducción Mara Glozman].

Presidencia de la Nación (1973). Plan Trienal para la Reconstrucción y la Liberación Nacional (1974-1977). Buenos Aires: PEN.

Programa Mundial de Empleo PME (1976). Empleo, crecimiento y necesidades esenciales: problema mundial. Génova: ILO.

Revel, J. (2008). El vocabulario de Foucault. Buenos Aires: Atuel.

Schwartz, R. (2014). Las ideas fuera de lugar. Meridional. Revista Chilena de Estudios Latinoamericanos, 3, 183-199.

Spicker, P., Alvarez Leguizamón, S., Gordon, D. (eds.) (2009). Necesidades Básicas. En Pobreza: Un glosario internacional (pp. 208-210). Buenos Aires: Consejo Latinoamericano de Ciencias Sociales.

Veyne, P. (1984). Foucault revoluciona la historia. En Cómo se escribe la Historia (pp. 199-238). Madrid: Alianza Editorial.

Walters, W. (2012). Reconecting governamentality and genealogy. Questions of style. En Governamentality. Critical encounters (pp. 110-140). New York: Routledge

Zavaleta, R. (1990). EI Estado en América Latina. En Obras completas, Tomo 3 (pp. 611-636). La Paz Cochabamba: Editorial Los Amigos del Libro.

\section{Notas}

1 Indagamos con mayor profundidad en este punto en Grondona, 2019a.

2 La noción de dominio interdiscursivo está inspirada en la perspectiva de Courtine (1981).

3 La frecuencia de publicación que nos exigen los sistemas de evaluación atenta contra estas lógicas de producción de más largo aliento. Por cierto, este modo de abordaje es también más afín al trabajo colectivo (que tampoco está muy estimulado), que permite que se vayan siguiendo varias pistas o hilos al mismo tiempo.

4 Nos referimos, por ejemplo, a los aportes de José Lezama Lima, Alejo Carpentier, Severo Sarduy o Bolívar Echeverría (Grondona, 2019a).

5 Cabe recordar, como dato que sirve para ilustrar este argumento, que la fase más violenta de la persecución racial bajo el régimen fascista se inició en 1938 con un texto que tuvo como (uno de sus) títulos Manifesto degli scienziati razzisti y que fue firmado por diez científicos. Del mismo modo, las declaraciones de UNESCO sobre las razas (en particular la de 1949 y 1951), en las que se establecía su inexistencia (en el caso de la primera declaración) o la improcedencia de establecer desigualdades objetivas entre ellas (para la segunda) fueron firmadas por una serie de científicos de renombre (del Norte y del Sur) convocados a tal efecto. 